\title{
(4) Serum Electrolytes
}

\author{
Eiichi KaTO \\ Department of Internal Medicine, Keio University, School of Medicine
}

Electrolytes are the key to homeostasis and furthermore their regulation is dependent upon renal function.

In acute renal failure cardiotoxicity due to hyperkalemia is the most fatal problem, however, coexisting acidosis and hyponatremia not only cause change in blood potassium level but also produce a direct influence on EKG (Fig. 1). Ion exchange

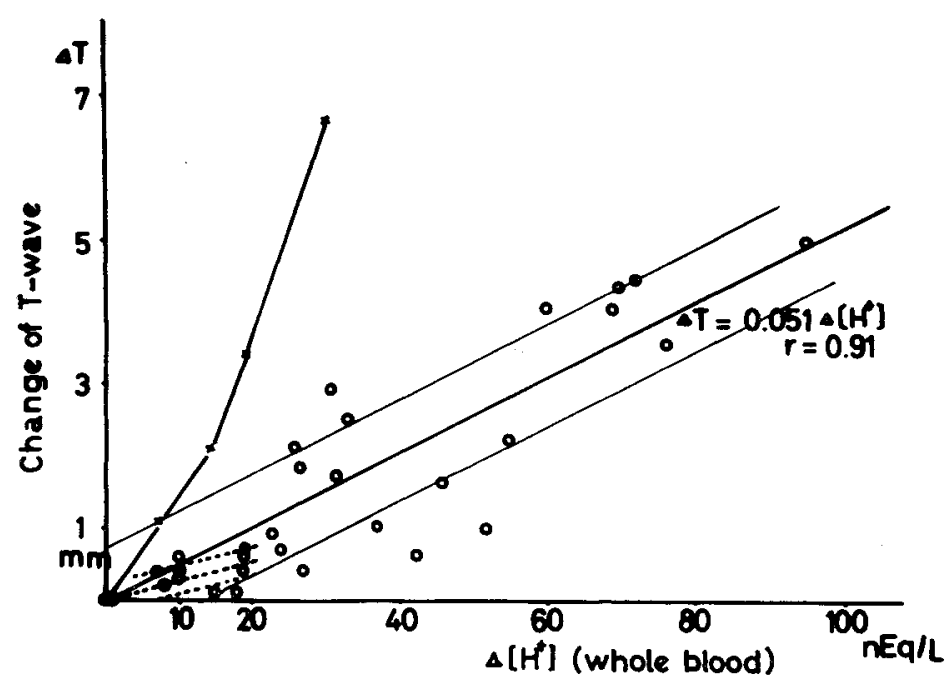

Fig. 1. The effect of respiratory acidosis on the T-wave of E.C.G.

resin, glucose with insulin are also effective, but artificial dialysis permits simultaneous correction of all these shifts of serum factors, and yet careful consideration has to be given to disequilibrium syndrome that may eventually occur following retarded "wash-out" of urea from the brain tissue through the brain-blood barrier.

In the meantime, prolonged life expectancy of patients with chronic renal failure has recently raised the much-discussed question of calcium metabolism. It would be meaningless to attempt to correct acidosis alone, because this may give rise to tetany or twitching. In some cases, acidosis, hypocalcemia, hyperphosphatemia, and an elevated level of BUN could be successfully improved following administration of $\mathrm{CaCO}_{3}$, while the most difficult feature is to determine the optimal dose.

Some believe that the use of Ringer's solution or physiological saline induces a 
severe acidosis, since these solutions contain more $\mathrm{Cl}^{-}$than $\mathrm{Na}^{+}$which would result in elimination of a considerable quantity of $\mathrm{HCO}_{3}^{-}$from the serum, which in its normal situation the ratio of $\mathrm{Na}^{+}$to $\mathrm{Cl}^{-}$is 140 to 100 .

However, we ${ }^{1)}$ conducted an experiment on dogs by intravenous administration of physiological saline, 5\% glucose solution (without $\mathrm{Cl}^{-}$) and $5 \%$ mannitol at the rate of $3.5 \mathrm{ml} . / \mathrm{kg}$. of body weight $/ \mathrm{min}$. As a result, nearly the same degree of $\mathrm{pH}$ fall was observed in each of these solutions; standard bicarbonate assay, considering respiratory factor, also showed the same decreasing tendency, while no remarkable change was noted in $\mathrm{PCo}_{2}$ (Fig. 2).

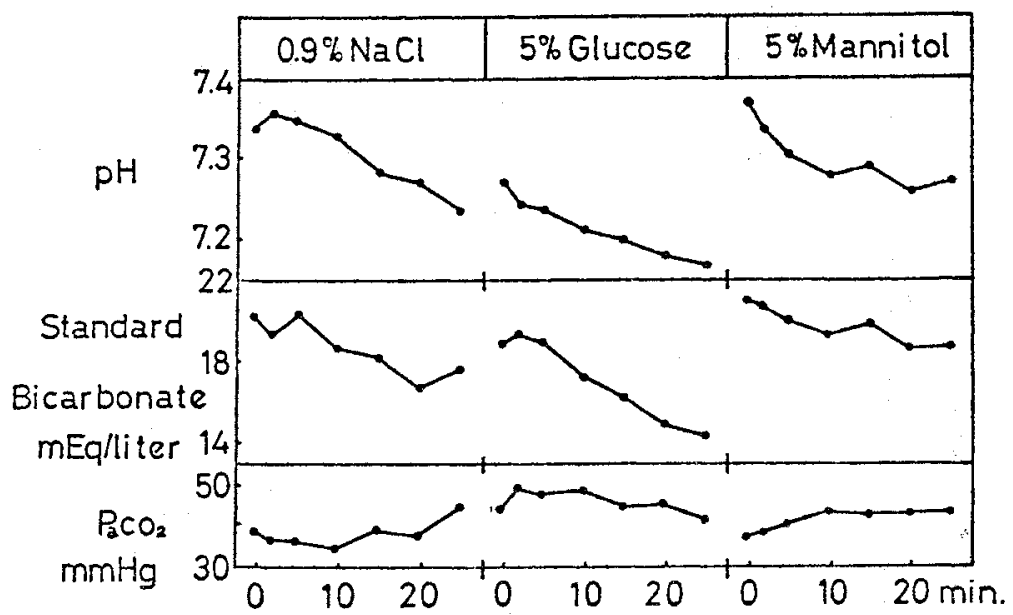

Fig. 2. Three representative examples of infusion of isotonic solution, showing the change of blood $\mathrm{pH}, \mathrm{PacO}_{2}$ and standard bicarbonate.

From the equation,

$$
\mathrm{pH}=6.1+\log -\frac{\mathrm{NaHCO}_{3}}{\mathrm{H}_{2} \mathrm{CO}_{3}}-
$$

the reason why acidosis provoked following administration of solutions with or without $\mathrm{Cl}^{-}$could be reasonably explained. It is the dilution of $\mathrm{HCO}_{3}^{-}$by the common solvent, water and not by its chloride content.

Maxwell and his group $^{2)}$. recently performed a similar experiment in which nephrectomized dogs were used to evaluate $\mathrm{pH}$ of the cellular fluid, and they demonstrated that our conclusion was reasonable, even though there was a minimal shift affecting the extra- and intracellular fluids.

At the same time, a simultaneous administration of $\mathrm{Cl}^{-}$frequently turns out to be clinically significant, as in the case when hypokalemia has to be corrected promptly.

In the renal tubular cells, the majority of $\mathrm{Na}^{+}$is reabsorbed together with $\mathrm{Cl}^{-}$ and the rest is exchanged by $\mathrm{K}^{+}$or $\mathrm{H}^{+}$. Both hypokalemia and lowered $\mathrm{pH}$ are difficult to correct in hypochloremic circumstances, because exchange of $\mathrm{Na}^{+}$by 
$\mathrm{K}^{+}$or $\mathrm{H}^{+}$is intensified. ${ }^{3)}$

The behavior of plasma $\mathrm{Cl}^{-}$reflects to a certain degree the state of $\mathrm{pH}$ regulation within the renal tubular cells. Thus, an increased level of plasma $\mathrm{Cl}^{-}$may be indicative of complicated pyelonephritis in renal insufficiency or of early signs of graft rejection in case of renal transplants.

The behavior of $\mathrm{Na}$ balance is closely related to that of the body fluid, especially to the volume of the extracellular fluid. According to the latest observations, natriuresis was observed by a large dose administration of physiological saline (saline diuresis), even under the lowered glomerular filtration rate (in renal artery compression procedure), or administration of a large dose of aldosterone. Consequently, it was presumed that the main site of those changes were most likely limited to the proximal renal tubulus and the change intermediated by the "third factor", which is now gradually becoming the focus of interest among world wide investigators.

In addition to the physical factors such as the renal interstitial volume or pressure, colloid osmotic pressure of peritubular capillaries, a humoral factor capable of directly blocking the renal tubulus may presumably be involved in this reabsorption suppression mechanism.

In our experiments with dogs, unilateral renal lymph vessels were ligated and some of the aforementioned physical factors were added. This resulted in an increased excretion from the kidney on this side in contrast to the contralateral kidney.

Using these dogs the following was also evaluated: 1) administration of physiological saline, 2) administration of physiological saline with chlorothiazide and ethacrynic acid (Fig. 3), 3) infusion of natriuretic plasma obtained from the dogs at the height of saline diuresis following administration of physiological saline, 4) control studies of normal plasma, 5) decreased excretion of $\mathrm{Na}$ due to compression procedure of the thoracic inferior vena cava, and 6) the change caused by injection of plasma obtained at that time.

The conclusion from this experiment was that physical factors are the most predominant, and eventual presence of natriuretic hormone was also undeniable. ${ }^{\text {) }}$

It has been reported that this

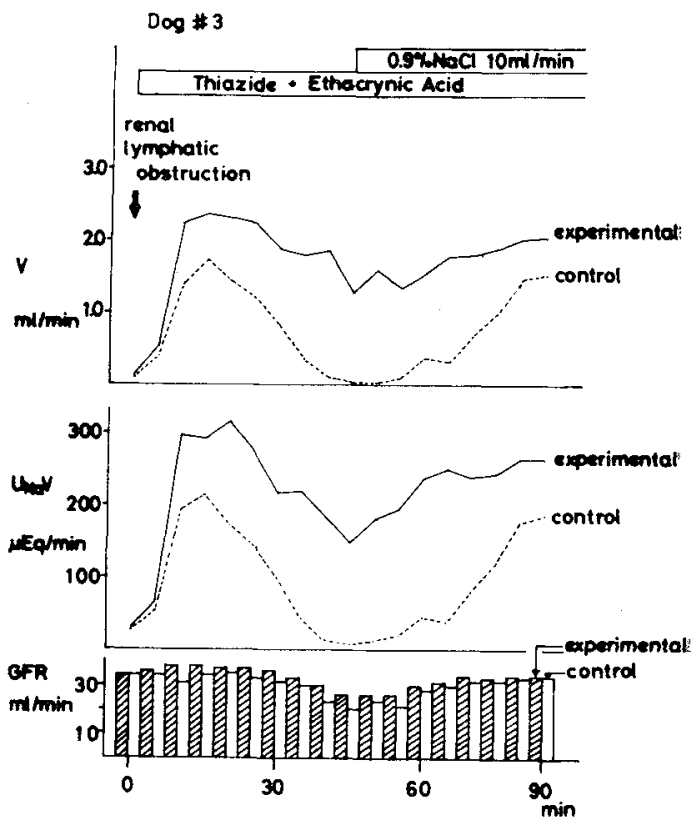

Fig. 3. The effect of renal lymphatic obstruction during distal blockade. 
mechanism may play certain role in escape phenomenon due to aldosterone, elevated natriuresis after salt load in hypertensive patients, and sodium losing tendency in patients with chronic renal failure. It was also indicated that sodium accumulation seen in edematous diseases may a part be the result of disturbance of this homeostatic mechanism.

Conclusions are as follows: 1) Homeostasis is maintained in a dynamic equilibrium with its several controlling factors intermediated by shift, and the factors are largely dependent upon each other. 2) There are two shifts existing, one is an unfavorable shift requiring immediate correction, and the other may be called a resetting or new steady state. 3) It is necessary to determine which shift is playing the leading role. As a rule, a gradual correction is needed until the patient's general condition improves, otherwise there is a chance of a shift to the opposite side. 4) Early recognition or prevention is most desirable, and abnormal serum chemistry itself is not the objective of therapy. First consideration must be given to the disease itself.

\section{References}

1) Asano, S. et al.: Lancent, $1: 1245,1966$.

2) Rosenbaum, B. J. et a1.: J. Lab. \& Clin. Med., 74:427, 1969.

3) Kassirer, J.P. and Schwartz, W. B.: Amer. J. Med., 40: 10, 1966.

4) Wada, $T$. et al.: Clin. Science, $38: 479,1970$. 